\title{
OTIMIZAÇÃO MULTIOBJETIVO PARA CONTROLE ROBUSTO APLICADO A INVERSORES CONECTADOS À REDE
}

\author{
Caio R. D. Osório ${ }^{1}$, Gustavo G. Koch ${ }^{1}$, Iury Cleveston ${ }^{1}$, Lucas C. Borin ${ }^{1}$, Fabrício H. Dupont ${ }^{2}$, \\ Ricardo C. L. F. Oliveira ${ }^{3}$, Vinícius F. Montagner ${ }^{1}$ \\ ${ }^{1}$ Universidade Federal de Santa Maria (UFSM), Santa Maria - RS, Brasil \\ ${ }^{2}$ Universidade Comunitária da Região de Chapecó (UNOCHAPECÓ), Chapecó - SC, Brasil \\ ${ }^{3}$ Universidade Estadual de Campinas (UNICAMP), Campinas - SP, Brasil \\ E-mail: caio.osorio@gmail.com
}

\begin{abstract}
Resumo - Este artigo propõe um novo procedimento de projeto para controladores por realimentação de estados com aplicação prática em inversores conectados à rede, levando a resultados em conformidade com a norma IEEE 1547, inclusive para o caso de implementação utilizando um número reduzido de sensores. $O$ procedimento baseiase na otimização de objetivos considerando estabilidade, erro de rastreamento e atenuação de distúrbios. Um algoritmo genético é utilizado para buscar, orientado por modelos simples, os ganhos de controle, levando a um conjunto de soluções viáveis (fronteira de Pareto). O ganho de controle escolhido possui estabilidade robusta contra incertezas e variações paramétricas certificada por meio de análise baseada em desigualdades matriciais lineares. Restrições no espaço de busca permitem encontrar, de forma automática, ganhos por realimentação total ou parcial de estados, superando limitações de desigualdades matriciais de síntese e levando a resultados experimentais de boa qualidade, o que torna este procedimento uma alternativa útil no projeto de controladores robustos para conversores de potência.
\end{abstract}

Palavras-chave - Algoritmos genéticos, Controle robusto, Desigualdades matriciais lineares, Inversores conectados à rede, Otimização multiobjetivo.

\section{MULTIOBJECTIVE OPTIMIZATION FOR ROBUST CONTROL APPLIED TO GRID-CONNECTED INVERTERS}

\begin{abstract}
This paper proposes a new design procedure for state feedback controllers suitable for practical application in grid-connected inverters, leading to results complying with IEEE 1547 Standard, even with a reduced number of sensors. The procedure relies on the optimization of objectives considering stability, tracking error and disturbance attenuation. A genetic algorithm is used to search, based on simple models, the control gains, leading to a set of viable solutions (Pareto front). The chosen control gain has robust stability against parameter uncertainties and variations certified by means of analysis linear matrix inequalities. Constraints in the search space allow to find full or partial state feedback
\end{abstract}

Artigo submetido em 26/09/2018. Primeira revisão em 24/11/2018. Aceito para publicação em 17/01/2019 por recomendação do Editor Marcello Mezaroba. http://dx.doi.org/10.18618/REP.2019.1.0041 gains in an automatic way, overcoming limitations of existing synthesis linear matrix inequalities and leading to experimental results of good quality, what makes this procedure a useful alternative on control synthesis for power converters.

Keywords - Genetic algorithms, Grid-connected inverters, Linear matrix inequalities, Multiobjective optimization, Robust control.

\section{INTRODUÇÃO}

Um ponto comum no projeto de controladores para conversores de potência é a necessidade de levar em consideração um bom compromisso entre diferentes objetivos, como respostas dinâmicas rápidas, rastreamento preciso, boa rejeição de distúrbios, robustez, sinal de controle limitado, etc. [1]. Uma forma de atingir esses objetivos é a utilização de otimização multiobjetivo para guiar o projeto de controladores. Desse modo, uma alternativa atraente é o uso de algoritmos genéticos (do inglês, Genetic Algorithms - GAs) [2]. Esses algoritmos podem encontrar soluções mesmo em espaços restritos de busca, sem depender das derivadas das funções objetivo, evoluindo também com base em simulações numéricas ou dados experimentais [2]. Além disso, GAs multiobjetivo podem fornecer um conjunto de soluções, conhecidas como fronteira de Pareto, permitindo que o projetista de controle tenha liberdade de escolher uma solução que possua um compromisso satisfatório entre os objetivos [1].

Para o controle de conversores de potência, por exemplo, em [3], um GA é usado para melhorar a resposta dinâmica de um conversor SEPIC por meio da otimização de um controlador de corrente PI usando um índice de erro de rastreamento na função objetivo. Em [4], um GA é utilizado para encontrar as matrizes de ponderação de um regulador quadrático linear discreto robusto (do inglês, Discrete Linear Quadratic Regulator - DLQR) aplicado a um conversor boost, em que uma função objetivo que leva em consideração o erro de rastreamento e o sinal de controle é proposta, visando otimizar a resposta dinâmica do sistema.

Entre as topologias de conversores de potência, os inversores conectados à rede são importantes no cenário de geração baseada em fontes renováveis, permitindo, por exemplo, controlar o fluxo de energia entre a fonte primária e a rede [5]. As funções básicas de controle para esta aplicação incluem controle de tensão do barramento $\mathrm{CC}$, sincronização 
com a rede e controle de corrente [6]. Para atenuar as harmônicas da modulação por largura de pulso (PWM) adotadas nos inversores, os filtros LCL são comumente aplicados. Neste contexto, o projeto de uma malha de controle robusto de corrente é particularmente importante, pois permite superar o problema de ressonância do filtro LCL, estabilizando o sistema em malha fechada, mesmo sob variação de impedância da rede e distúrbios de tensão da rede, garantindo correntes injetadas na rede que respeitem limites rigorosos de distorção harmônica [7]. Dentre as estratégias de controle usuais, podem ser citadas aquelas baseadas no domínio da frequência, como a estratégia proporcionalressonante, que impõe referências senoidais para as correntes harmônicas, e a estratégia PI nas coordenadas $d q$, que impõe referências constantes. Estratégias baseadas no domínio do tempo, como controladores por realimentação de estados, também podem ser destacadas [8]-[10].

Considerando a aplicação de GAs no controle de correntes de inversores conectados à rede, em [11] um GA é usado para otimizar constantes de um controlador PI. A função objetivo, neste caso, contém especificações de resposta transitória e erro em regime permanente. Em [12], o GA é utilizado para um projeto otimizado de controladores proporcionais-ressonantes e em [13] para o projeto do amortecimento ativo de filtros LCL. Analisando-se a literatura, é possível observar que existe um número reduzido de trabalhos que tratam de GAs para o projeto de controladores em conversores de potência, especialmente incluindo comprovação teórica de estabilidade e validação experimental, o que motiva novas investigações nessa direção [14].

Em relação ao projeto de controladores robustos, as desigualdades matriciais lineares (do inglês, Linear Matrix Inequalities - LMIs) são uma ferramenta bem estabelecida, especialmente para sistemas sujeitos a incertezas [15]. LMIs foram aplicadas com sucesso na prática no controle robusto de conversores de potência em vários trabalhos, como, por exemplo, em [16]. Apesar dos avanços significativos no contexto de análise, as condições de síntese baseadas em LMIs são suficientes e o nível de conservadorismo aumenta com o número de variáveis de estado e de parâmetros incertos. Além disso, em muitos casos, as condições de síntese podem levar a altos ganhos de controle ao otimizar uma única função objetivo, o que pode tornar a implementação experimental do controlador impraticável [17]. Outra tarefa desafiadora de projeto é a dificuldade de impor restrições nos elementos do ganho de controle, já que este raramente é uma variável de otimização (mas obtido a posteriori via mudança de variáveis) do problema [15]. Existem condições suficientes, mas, em geral, limitando a norma do ganho (e não os elementos do ganho), introduzindo mais conservadorismo [18]. Por outro lado, as condições de análise de estabilidade robusta baseadas em LMIs têm recebido significativos aprimoramentos. Atualmente existem condições necessárias e suficientes para análise de estabilidade robusta [19] e as mesmas podem ser úteis para certificar a estabilidade robusta dos controladores aplicados a conversores de potência.

Tendo em vista o exposto, a principal contribuição deste artigo é um procedimento de projeto para controladores por realimentação de estados aplicados a conversores de potência, combinando a capacidade de GAs encontrarem ganhos em um espaço de busca limitado com a capacidade de LMIs para certificar a estabilidade robusta do sistema em malha fechada frente a incertezas e variações paramétricas. $\mathrm{O}$ uso de GAs permite projetar ganhos fixos de controle que podem não ser obtidos por meio de LMIs de síntese atuais sem aumento significativo do grau de conservadorismo [15], [20]. O procedimento proposto é resumido na Figura 1, mostrando que a otimização multiobjetivo usada aqui é flexível, podendo levar em conta diferentes índices de desempenho, nos domínios da frequência e do tempo. A estabilidade robusta do sistema de malha fechada é certificada por meio de uma função Lyapunov obtida a partir da solução de um problema baseado em LMI. O procedimento é aplicado com sucesso ao controle de corrente de inversores trifásicos conectados à rede por meio de filtros LCL. A flexibilidade da abordagem proposta é confirmada para um projeto considerando uma realimentação parcial de estados, que permite implementar o controlador, na prática, com um número reduzido de sensores.

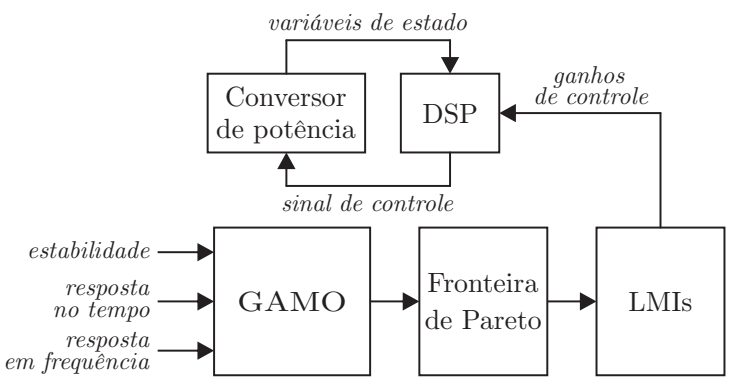

Fig. 1. Visão geral do procedimento de projeto proposto, combinando algoritmo genético multiobjetivo (GAMO) e LMIs.

\section{INVERSOR CONECTADO À REDE}

Considere o inversor conectado à rede por meio do filtro LCL, na Figura 2. Supõe-se que o sincronismo com a tensão do ponto de acoplamento comum (PCC) já esteja garantida e que a tensão de entrada $V_{c c}$ esteja devidamente estabilizada [6]. A indutância da rede, $L_{g}$, é assumida como um parâmetro incerto, pertencente ao intervalo dado por $L_{g} \in \mathscr{U}, \mathscr{U}=\left\{L_{g} \in \mathfrak{R}^{+}: L_{g \min } \leq L_{g} \leq L_{\text {gmax }}\right\}$.

A planta na Figura 2 pode ser representada, no referencial estacionário, por dois modelos desacoplados no espaço de estados [21]

$$
\begin{aligned}
& {\left[\begin{array}{c}
\dot{\boldsymbol{x}}_{\alpha} \\
\dot{\boldsymbol{x}}_{\beta}
\end{array}\right]=\left[\begin{array}{cc}
\boldsymbol{A}\left(L_{g}\right) & \mathbf{0}_{3 \times 3} \\
\mathbf{0}_{3 \times 3} & \boldsymbol{A}\left(L_{g}\right)
\end{array}\right]\left[\begin{array}{c}
\boldsymbol{x}_{\alpha} \\
\boldsymbol{x}_{\beta}
\end{array}\right]+} \\
& {\left[\begin{array}{cc}
\boldsymbol{B}_{u} & \mathbf{0}_{3 \times 1} \\
\mathbf{0}_{3 \times 1} & \boldsymbol{B}_{u}
\end{array}\right]\left[\begin{array}{c}
u_{\alpha} \\
u_{\beta}
\end{array}\right]+\left[\begin{array}{cc}
\boldsymbol{B}_{d}\left(L_{g}\right) & \mathbf{0}_{3 \times 1} \\
\mathbf{0}_{3 \times 1} & \boldsymbol{B}_{d}\left(L_{g}\right)
\end{array}\right]\left[\begin{array}{c}
v_{g \alpha} \\
v_{g \beta}
\end{array}\right],}
\end{aligned}
$$

com

$$
\begin{gathered}
\boldsymbol{A}\left(L_{g}\right)=\left[\begin{array}{ccc}
0 & -\frac{1}{L_{c 1}} & 0 \\
\frac{1}{C_{f}} & 0 & -\frac{1}{C_{f}} \\
0 & \frac{1}{L_{c 2}+L_{g}} & 0
\end{array}\right], \boldsymbol{B}_{u}=\left[\begin{array}{c}
\frac{1}{L_{c 1}} \\
0 \\
0
\end{array}\right] \\
\boldsymbol{B}_{d}\left(L_{g}\right)=\left[\begin{array}{c}
0 \\
0 \\
-\frac{1}{L_{c 2}+L_{g}}
\end{array}\right], \boldsymbol{x}_{\alpha}=\left[\begin{array}{l}
i_{c \alpha} \\
v_{c \alpha} \\
i_{g \alpha}
\end{array}\right], \boldsymbol{x}_{\beta}=\left[\begin{array}{c}
i_{c \beta} \\
v_{c \beta} \\
i_{g \beta}
\end{array}\right] .
\end{gathered}
$$




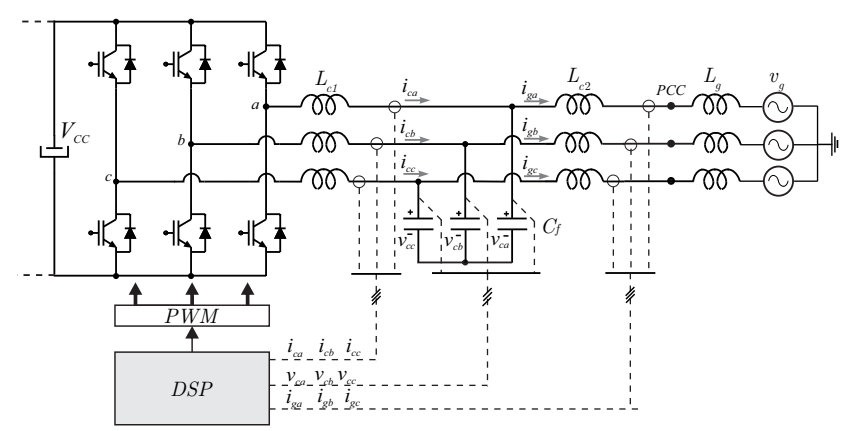

Fig. 2. Inversor trifásico conectado à rede por meio de um filtro LCL.

Em (2), para as coordenadas $\alpha$ e $\beta$, os estados $i_{c}, v_{c}$ e $i_{g}$ representam, respectivamente, a corrente no indutor do lado do conversor, a tensão no capacitor do filtro e a corrente injetada na rede, $u$ representa a entrada de controle e $v_{g}$ a tensão da rede. Por simplicidade de notação, a partir deste ponto, os subscritos $\alpha$ e $\beta$ serão suprimidos, sendo a representação válida para ambos os eixos.

Para a aplicação de uma lei de controle digital, considere a discretização da planta com um período de amostragem suficientemente pequeno, $T_{s}$, e também a inclusão de um estado adicional, $\phi$, representando o atraso na implementação do sinal de controle [22], levando a

$$
\begin{aligned}
& \boldsymbol{x}(n+1)=\boldsymbol{A}_{d}\left(L_{g}\right) \boldsymbol{x}(n)+\boldsymbol{B}_{u d}\left(L_{g}\right) \boldsymbol{\phi}(n)+\boldsymbol{B}_{d d}\left(L_{g}\right) v_{g}(n), \\
& \phi(n+1)=u(n)
\end{aligned}
$$

em que o subscrito $d$ denota matrizes discretas obtidas a partir das apresentadas em (2) [16].

Para garantir o rastreamento de referências senoidais e rejeição de harmônicas, os controladores ressonantes

$$
\boldsymbol{\xi}(n+1)=\boldsymbol{R} \boldsymbol{\xi}(n)+\boldsymbol{T}\left(i_{r e f}(n)-i_{g}(n)\right)
$$

são incluídos no modelo [23]. Em (4), assumindo que $r$ é o número de controladores ressonantes, $\boldsymbol{\xi}$ é um vetor contendo $2 r$ estados internos, sendo $\boldsymbol{R}$ e $\boldsymbol{T}$ as matrizes associadas aos controladores ressonantes, com dimensão $(2 r \times 2 r)$ e $(2 r \times 1)$, respectivamente. Mais detalhes sobre estes controladores podem ser vistos, por exemplo, na Seção 2 de [16]. O sinal $i_{\text {ref }}$ representa a referência para as correntes da rede $\left(i_{g}\right)$, no eixo $\alpha$ ou $\beta$.

O modelo (3) pode ser reescrito, com o vetor de estados aumentado dado por $\boldsymbol{\rho}(n)=[\boldsymbol{x}(n) \boldsymbol{\phi}(n) \boldsymbol{\xi}(n)]^{\prime}$

$$
\begin{aligned}
& \boldsymbol{\rho}(n+1)=\boldsymbol{A}_{\text {aug }}\left(L_{g}\right) \boldsymbol{\rho}(n)+\boldsymbol{B}_{\text {uaug }} u(n)+\boldsymbol{B}_{\text {daug }}\left(L_{g}\right) v_{g}(n) \\
& +\boldsymbol{B}_{\text {raug }} i_{\text {ref }}(n) \text {, } \\
& y(n)=\boldsymbol{C}_{a u g} \boldsymbol{\rho}(n)=i_{g}(n), \boldsymbol{C}_{a u g}=\left[\begin{array}{llllll}
0 & 0 & 1 & 0 & 0_{1 \times 2 r}
\end{array}\right],
\end{aligned}
$$

em que $\boldsymbol{\rho}$ tem dimensão $(4+2 r)$ e as entradas $u, v_{g}$ e $i_{r e f}$ são escalares.

De um modo geral, o problema do controle de corrente a ser resolvido aqui é sintetizar a entrada de controle $u$ (que após modulação adequada equivale a média das tensões de saída do inversor PWM) para assegurar que as correntes da rede rastreiem referências senoidais, sob indutância de rede incerta. A formulação de um problema de controle específico para atender a esses objetivos é dada na próxima seção.

\section{FORMULAÇÃO DO PROBLEMA MULTIOBJETIVO}

Considere, para cada coordenada $\alpha$ ou $\beta$, a lei de controle por realimentação de estados dada por

$$
u(n)=\boldsymbol{K} \boldsymbol{\rho}(n)=\left[\begin{array}{lll}
\boldsymbol{K}_{x} & K_{\phi} & \boldsymbol{K}_{\xi}
\end{array}\right]\left[\begin{array}{l}
\boldsymbol{x}(n) \\
\boldsymbol{\phi}(n) \\
\boldsymbol{\xi}(n)
\end{array}\right]
$$

Neste artigo, o objetivo é calcular, off-line, o ganho de controle fixo, $\boldsymbol{K}=\left[\begin{array}{lll}\boldsymbol{K}_{x} & K_{\phi} & \boldsymbol{K}_{\xi}\end{array}\right]$, para: i) garantir estabilidade robusta contra incertezas em $L_{g} \in \mathscr{U}$, ii) assegurar rastreamento de referências senoidais de correntes da rede, iii) atenuar harmônicas de corrente da rede a partir das tensões de saída PWM do inversor.

Adota-se, neste trabalho, o mesmo ganho de controle para as coordenadas $\alpha$ e $\beta$ [24]. Isto é possível pois assume-se sistema trifásico equilibrado e, portanto, eixos desacoplados e com representação idêntica, como em (1).

A propriedade i) pode ser atingida por meio da minimização da função objetivo

$$
\boldsymbol{\sigma}(\boldsymbol{K})=\max _{L_{g} \in \mathscr{U}}\left|\lambda\left(\boldsymbol{A}_{\text {aug }}\left(L_{g}\right)+\boldsymbol{B}_{\text {uaug }} \boldsymbol{K}\right)\right|,
$$

que fornece o máximo módulo dos autovalores do sistema em malha fechada.

A propriedade ii) pode ser atingida por meio da minimização de uma função objetivo baseada na integral do erro quadrático (do inglês, Integral Squared Error - ISE), dado por

$$
\varepsilon(\boldsymbol{K})=\max _{L_{g} \in \mathscr{U}}\left(\frac{1}{N_{2}-N_{1}} \sum_{n=N_{1}}^{N_{2}} e^{2}(n)\right),
$$

em que o erro de rastreamento é escrito como

$$
e(n)=i_{r e f}(n)-i_{g}(n) .
$$

Os números $N_{1}$ e $N_{2}$ são inteiros escolhidos para definir as amostras inicial e final para o cálculo desse erro.

A propriedade iii) pode ser atingida por meio da minimização da função objetivo

$$
\gamma(\boldsymbol{K})=\max _{L_{g} \in \mathscr{U}}\left|\boldsymbol{C}_{\text {aug }}\left(e^{j \omega T_{s}} I-\left(\boldsymbol{A}_{\text {aug }}\left(L_{g}\right)+\boldsymbol{B}_{\text {uaug }} K\right)\right)^{-1} \boldsymbol{B}_{\text {uaug }}\right|
$$

assumindo para (10) uma entrada na forma de distúrbio aditivo no sinal $u$. Esta é uma métrica obtida no domínio da frequência, em que $\omega$ é um número real, pertencente ao intervalo $\left[0,2 \pi / T_{s}\right]$. Esta função leva em consideração a atenuação de harmônicas das tensões de saída do inversor PWM para a corrente de saída $i_{g}$. Tais harmônicas têm origem em não-linearidades, como por exemplo o tempo morto no acionamento dos interruptores.

A partir das funções objetivo estabelecidas, o problema de projeto do controlador pode ser expresso por meio de um problema de otimização multiobjetivo, dado por

$$
\begin{aligned}
& \min (\sigma(\boldsymbol{K}), \varepsilon(\boldsymbol{K}), \gamma(\boldsymbol{K})), \\
& \text { s.a } \boldsymbol{K} \in \mathscr{K},
\end{aligned}
$$

em que $\mathscr{K}$ é o espaço de busca para os ganhos de controle, que deve ser definido pelo projetista. 
Uma vantagem de abordar problemas multiobjetivo com funções específicas para cada objetivo é permitir que o projetista encontre um conjunto de soluções nas quais um compromisso entre os objetivos possa ser investigado.

Para ilustrar a eficácia do método proposto, este trabalho considerará, para um estudo de caso, os parâmetros da Tabela I, de [24]. Foram considerados 4 controladores ressonantes $(r=4)$, sintonizados na frequência fundamental da tensão de rede e suas três primeiras harmônicas ímpares, assumindo que a rede é predominantemente distorcida por estas harmônicas [16].

\section{TABELA I}

Parâmetros do Sistema

\begin{tabular}{cc}
\hline Parâmetros & Valor \\
\hline Parâmetro do Filtro $L_{c 1}, C_{f}$ e $L_{c 2}$ & $1 \mathrm{mH}, 62 \mu \mathrm{F} \mathrm{e} 0,3 \mathrm{mH}$ \\
\hline Indutância da Rede $\left[L_{g \min }, L_{g \max }\right]$ & {$[0,1] \mathrm{mH}$} \\
\hline Tensão Barramento $-V_{c c}$ & $400 \mathrm{~V}$ \\
\hline Tensão da Rede RMS & $127 \mathrm{~V} / 60 \mathrm{~Hz}$ \\
\hline Frequência de Amostragem & $20040 \mathrm{~Hz}$ \\
\hline Frequência de Comutação & $10020 \mathrm{~Hz}$ \\
\hline Frequências dos controladores ressonantes & $60,180,300,420 \mathrm{~Hz}$. \\
\hline Fator de amortecimento & 0,0001
\end{tabular}

Note que, neste caso, o ganho $\boldsymbol{K}$ em (6) tem 12 elementos, relativos à realimentação de 3 estados do filtro, $\boldsymbol{x}$, ao estado do controle atrasado, $\phi$, e a 8 estados associados aos controladores ressonantes, $\boldsymbol{\xi}$. O ganho $\boldsymbol{K}$ é dado por

$$
\boldsymbol{K}=\left[\begin{array}{llll}
k_{1} & k_{2} & \ldots & k_{12}
\end{array}\right]
$$

com elementos pertencentes a $\mathscr{K}$, representado por

$$
\mathscr{K}=\left\{\boldsymbol{K} \in \mathfrak{R}^{12}: \boldsymbol{K}_{\text {inf }} \leq \boldsymbol{K} \leq \boldsymbol{K}_{\text {sup }}\right\} .
$$

A escolha dos limites inferior e superior, $\boldsymbol{K}_{\text {inf }}$ e $\boldsymbol{K}_{\text {sup }}$, respectivamente, permite restringir o espaço de busca. Por exemplo, para projetar um controlador por realimentação parcial de estados, o ganho associado a um dos estados pode ser definido como zero, o que é feito facilmente definindo como zero os limites da respectiva entrada.

É importante mencionar que, mesmo considerando apenas a estabilização robusta do sistema $(\sigma(\boldsymbol{K})<1)$, não existem na literatura condições necessárias e suficientes baseadas em LMIs. A razão para esse fato é a necessidade de impor restrições de estrutura na matriz de Lyapunov ou nas variáveis de folga para linearizar as desigualdades. Ainda, note que a função objetivo (8) tem $K$ como parâmetro de entrada, ou seja, é um critério avaliado no tempo e só pode ser calculado após o ganho $K$ ser determinado. Consequentemente, combinar $\sigma(\boldsymbol{K}), \varepsilon(\boldsymbol{K})$ e $\gamma(\boldsymbol{K})$, em (11), impossibilita a formulação do problema com LMIs de síntese de forma a recuperar os ganhos a partir de uma transformação matricial.

\section{PROJETO DE CONTROLE BASEADO EM GA MULTIOBJETIVO E LMI}

Para resolver o problema de projeto de controle descrito na seção anterior, o algoritmo NSGA-II foi escolhido devido à sua baixa complexidade computacional e capacidade de encontrar uma boa dispersão de soluções, além de apresentar uma boa convergência perto da fronteira de Pareto ótima [25].
Aqui, esse algoritmo é configurado para gerar aleatoriamente a população inicial, na qual cada indivíduo é descrito por um vetor de ganho $\boldsymbol{K}$. Por exemplo, para $\boldsymbol{K}$ em (12), cada indivíduo contém 12 genes. Os valores das funções objetivo (7), (8) e (10) são computados para cada indivíduo e, em seguida, estes são classificados considerando-se como melhor aquele que obteve o menor valor na respectiva função. A próxima geração de indivíduos é formada pela aplicação de seleção, cruzamento, mutação e elitismo na geração atual. Então, uma fronteira de Pareto é obtida. Cabe observar que, para a obtenção da fronteira de Pareto final, são eliminados os indivíduos com $\sigma(\boldsymbol{K})>1$, por serem sabidamente instáveis. Finalmente, baseado na norma-2 (norma euclidiana) das funções objetivo, um indivíduo é escolhido nessa fronteira de modo a garantir um bom compromisso entre os objetivos [2].

O GA multiobjetivo é uma alternativa interessante, pois permite definir o espaço de busca para o ganho de controle, e as funções objetivo podem ser determinadas facilmente apenas com operações algébricas matriciais, neste caso. Uma desvantagem neste ponto é que a análise de estabilidade baseada em (7) considera valores discretos para $L_{g}$, exigindo uma prova teórica de estabilidade robusta para todos os valores de $L_{g} \in \mathscr{U}$. Neste sentido, diferentemente de trabalhos sobre GA aplicado ao controle de conversores conectados à rede [11]-[13], aqui a certificação de estabilidade para este ganho de controle é incluída no procedimento de projeto, conforme destacado no fluxograma da Figura 3. Esta etapa é realizada no final da execução do NSGA-II e é baseada no fornecimento de uma função Lyapunov como um certificado de estabilidade, obtido por meio de LMIs, que garante robustez mesmo contra variações paramétricas arbitrárias de $L_{g} \in \mathscr{U}$. Caso o algoritmo não produza fronteira de Pareto final com indivíduos viáveis ou caso o indivíduo selecionado não tenha estabilidade certificada, os parâmetros do GA devem ser ajustados e o algoritmo executado novamente.

Para o estudo de caso apresentado aqui, o algoritmo NSGAII foi inicializado com uma população de 500 indivíduos gerados aleatoriamente usando distribuição uniforme, com os limites superior e inferior em (13) dados por

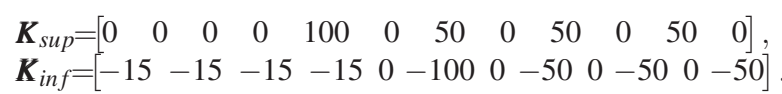

Os limites apresentados acima foram escolhidos em torno

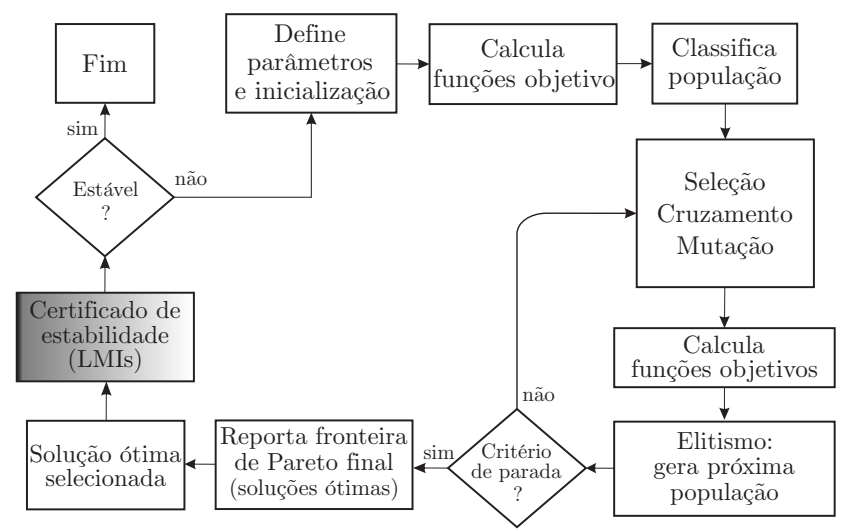

Fig. 3. Fluxograma baseado no NSGA-II, incluindo a fronteira de Pareto final, a seleção de uma solução ótima e a certificação de estabilidade baseada em LMIs. 
dos ganhos de um DLQR [26] convencional, projetado com os parâmetros da Tabela I, considerando $L_{g}=0,5 \mathrm{mH}$ como valor nominal, com matrizes de ponderação escolhidas como unitárias, por simplicidade.

Para calcular os valores da função objetivo (7), foi utilizada uma discretização fina no intervalo $\left[L_{\text {gmin }}, L_{\text {gmax }}\right]$, visto na Tabela I, e para calcular as funções objetivo (8) e (10), apenas os valores extremos foram empregados. Como a população é inicializada aleatoriamente, nas primeiras gerações há indivíduos com respostas instáveis, que são identificadas quando $\sigma(\boldsymbol{K})$ é maior que um. Nessa situação, a simulação para o cálculo de $\varepsilon(\boldsymbol{K})$ resultaria em um valor numérico não válido e, portanto, para evitar o consumo de tempo em simulação com indicativo de instabilidade, $\varepsilon(\boldsymbol{K})=10^{6}$ foi atribuído a esses indivíduos, penalizando-os no processo de seleção para as gerações futuras.

É importante notar que seria possível (usando LMIs, por exemplo) gerar uma população inicial formada apenas com indivíduos estáveis. No entanto, devido a cruzamentos e mutações, não haveria garantias de que a estabilidade da população inicial seria transmitida para a prole durante a evolução do algoritmo. Portanto, o problema persistiria, a menos que uma verificação de estabilidade fosse realizada para todos os indivíduos em cada geração, o que aumentaria a carga computacional. Além disso, a aleatoriedade da população inicial permite uma boa diversidade e, portanto, uma ampla busca no hiperplano da solução. Neste sentido, este trabalho propõe como contribuição uma metodologia de projeto baseada em GA com uma certificação a posteriori de estabilidade robusta por meio de LMIs, que pode oferecer uma conclusão exata sobre a estabilidade robusta do sistema de malha fechada. Esta certificação é calculada somente quando os critérios de parada do GA são atendidos, evitando assim a carga computacional excessiva.

Em relação às configurações do NSGA-II, a evolução é realizada por meio do algoritmo MuplusLambda, em que a seleção é baseada no elitismo, adicionando indivíduos da população atual $(\mu)$ aos melhores indivíduos da população anterior $(\lambda)$, produzindo uma população com tamanho $(\mu+$ $\lambda)$ que garante a sobrevivência do melhor indivíduo. $\mathrm{O}$ método de cruzamento usado foi Simulated Binary Bounded, com uma taxa de $80 \%$. O método de mutação usado foi o Polynomial Bounded, com uma taxa de $20 \%$ (veja [25] para detalhes sobre as configurações do algoritmo). O critério de parada foi a estagnação, caracterizada por 25 gerações sem mudanças significativas nas funções objetivo (reduções inferiores a $10^{-4}$ ). A Tabela II resume os parâmetros usados pelo GA.

TABELA II

Configurações do GA

\begin{tabular}{cc}
\hline Tamanho da População & 500 \\
\hline Taxa de Cruzamento & $80 \%$ \\
\hline Taxa de Mutação & $20 \%$ \\
\hline Método de Seleção & NSGA-II \\
\hline Elitismo & Sim \\
\hline Método de Cruzamento & Bounded Binary Crossover \\
\hline Método de Mutação & Bounded Polynomial Mutation \\
\hline Estratégia de Evolução & População $\mu+\lambda$ \\
\hline Critério de Parada & 25 gerações com estagnação \\
\hline
\end{tabular}

O procedimento na Figura 3 foi executado várias vezes com os parâmetros descritos anteriormente e sempre convergiu, produzindo fronteiras de Pareto finais nas quais o indivíduo selecionado teve a estabilidade certificada. A fronteira de Pareto final para uma das execuções é mostrada na Figura 4, detalhando o indivíduo selecionado, que fornece a menor norma-2 das funções objetivo. Este será o indivíduo utilizado no principal exemplo a ser detalhado ao longo deste artigo.

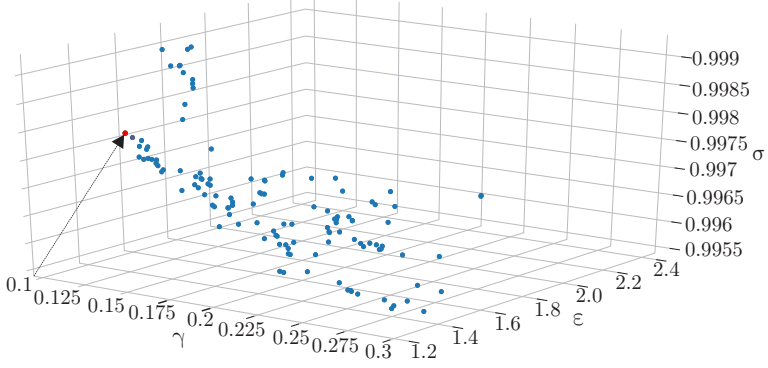

Fig. 4. Indivíduo selecionado na fronteira de Pareto final, apresentando a menor norma-2.

A solução selecionada (indicada por uma seta na Figura 4) tem os valores da função objetivo $(\sigma, \varepsilon$ e $\gamma)$ e os ganhos de controle dados por (15). Para confirmar essas métricas associadas a este ganho $\boldsymbol{K}$, testes de simulação específicos foram realizados para o sistema de malha fechada.

$$
\begin{aligned}
& \sigma=0,99778 \quad \varepsilon=1,4712 \quad \gamma=0,11578
\end{aligned}
$$

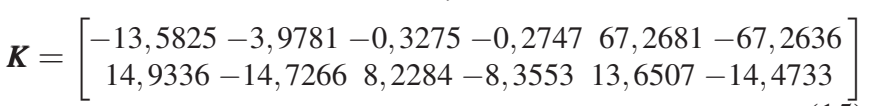

A Figura 5.a mostra as formas de onda de referência e de saída, assim como o sinal de erro associado, para a simulação com $L_{g}=L_{\text {gmax }}$ (pior caso), confirmando o valor $\varepsilon=1,4712$. Este teste confirma o bom rastreamento do sinal de referência, incluindo variações de fase e amplitude para representar a injeção de potência reativa (indutiva e capacitiva) e ativa na rede. A Figura 5.b mostra a resposta em frequência da entrada $u$ para a saída $i_{g}$, considerando valores de $L_{g} \in \mathscr{U}$. Observe a atenuação para todas as frequências e para todos os valores de $L_{g}$. No pior caso, o valor do ganho é dado por $-18,7860 \mathrm{~dB}$, que, convertido em valor absoluto, resulta em $\gamma=0,11578$, confirmando a métrica em (15) para o controlador.

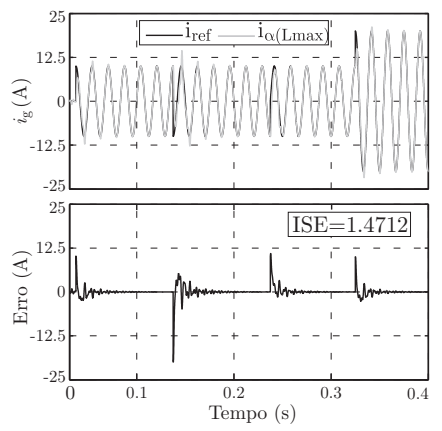

(a)

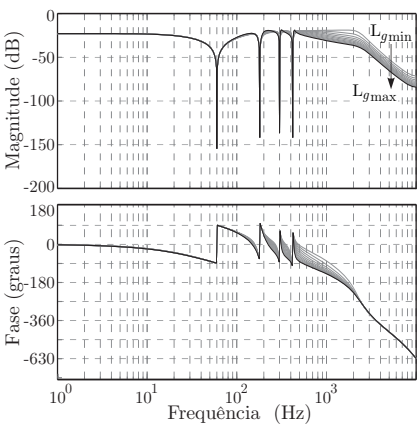

(b)
Fig. 5. (a) Simulação de rastreamento de referência para o sistema em malha fechada com o ganho (15), para $L_{g}=L_{\text {gmax }}$. (b) Diagramas de Bode de $u$ para $i_{g}$, confirmando o valor, no pior caso, de $\gamma=0,11578$. 


\section{A. Certificado de Estabilidade Robusta sob Variações Paramétricas da Rede}

Um ganho de controle que garante um valor $\sigma<1$ indica estabilidade robusta para $L_{g}$ incerto mas invariante no tempo. Entretanto, o cálculo de $\sigma$ durante a otimização é realizado para valores discretos de $L_{g} \in \mathscr{U}$. Quanto mais fina a discretização no intervalo $\mathscr{U}$, mais precisa é a conclusão sobre a estabilidade, mas ao preço de aumentar significativamente o tempo computacional requerido nesta análise. Por este motivo, neste trabalho foi escolhida uma discretização em $L_{g}$ suficiente para fornecer uma boa indicação de estabilidade, sem aumentar significativamente o tempo computacional enquanto o NSGA-II está evoluindo. Neste sentido, um certificado teórico de estabilidade para qualquer valor de $L_{g} \in \mathscr{U}$, incluindo possíveis variações paramétricas em $L_{g}$ (lentas ou arbitrariamente rápidas), é uma questão importante e foi adicionado a posteriori ao procedimento de projeto proposto na Figura 3, como uma contribuição deste artigo.

Considere o sistema (5) operando com o controlador (6), com $L_{g}$ assumindo qualquer valor em $L_{g} \in \mathscr{U}$ em qualquer instante de tempo. A estabilidade em malha fechada, neste caso, pode ser analisada por meio da estabilidade assintótica do sistema politópico [15]

$$
\boldsymbol{\rho}(n+1)=\boldsymbol{A}_{c l}(\theta(n)) \boldsymbol{\rho}(n),
$$

com

$$
\boldsymbol{A}_{c l}(\boldsymbol{\theta}(n))=\theta(n) \boldsymbol{A}_{c l 1}+(1-\theta(n)) \boldsymbol{A}_{c l 2}
$$

para todos os reais $\theta(n)$, com $0 \leq \theta(n) \leq 1$, e com os vértices dado por

$$
\boldsymbol{A}_{c l 1}=\boldsymbol{A}_{\text {aug }}\left(L_{g \text { min }}\right)+\boldsymbol{B}_{\text {uaug }} \boldsymbol{K}
$$

$\mathrm{e}$

$$
\boldsymbol{A}_{c l 2}=\boldsymbol{A}_{\text {aug }}\left(L_{\text {gmax }}\right)+\boldsymbol{B}_{\text {uaug }} \boldsymbol{K} .
$$

O sistema variante no tempo (16) é assintoticamente estável se existe uma matriz $\boldsymbol{P}=\boldsymbol{P}^{\prime}>0$ de tal modo que [15]

$$
\boldsymbol{A}_{c l i}^{\prime} \boldsymbol{P} \boldsymbol{A}_{c l i}-\boldsymbol{P}<0, i=1,2 .
$$

Este resultado é baseado em uma função Lyapunov dada por $v(\boldsymbol{\rho}(n))=\boldsymbol{\rho}(n)^{\prime} \boldsymbol{P} \boldsymbol{\rho}(n)$, que certifica a estabilidade do sistema em malha fechada para variações de parâmetro lentas ou arbitrariamente rápidas.

Verificando a factibilidade de (20) para os vértices (18) e (19), com o ganho de controle (15), existe solução $\boldsymbol{P}$ para o problema, que certifica a estabilidade do sistema para $L_{g} \in$ $\mathscr{U}$ incerto e invariante no tempo e também para $L_{g}$ variando arbitrariamente no tempo dentro deste conjunto.

Considerando os bons resultados de simulação e o certificado de estabilidade fornecidos nesta seção, a viabilidade experimental do ganho (15) é verificada experimentalmente na sequência.

\section{RESULTADOS EXPERIMENTAIS}

Para a implementação prática dos controladores projetados por meio do GA multiobjetivo, utilizou-se a configuração experimental mostrada na Figura 6, composta por um inversor trifásico baseado em IGBTs e um filtro LCL trifásico conectado à rede. Esta configuração tem uma potência nominal de $5,4 \mathrm{~kW}$, e os parâmetros dados na Tabela I. Os estados do filtro (correntes e tensões) são obtidos usando sensores de efeito Hall. A lei de controle é sintetizada em um DSP TMS320F28335 (Texas Instruments). A sincronização com a rede no PCC é fornecida por um algoritmo de filtro de Kalman [27].

O primeiro teste experimental realizado com o sistema em malha fechada controlado pelo ganho (15) diz respeito ao rastreamento de referência considerando as variações de fase e amplitude mostradas na Figura 5 .a. Estas variações nas referências em $\alpha$ e $\beta$ correspondem a injeção de potência reativa (indutiva ou capacitiva) e potência ativa na rede, respeitando a defasagem de $90^{\circ}$ entre os eixos. Os resultados para o eixo $\alpha$ são mostrados nas Figuras 7.a) e Figuras 7.b, que ilustram, respectivamente, uma simulação numérica baseada no modelo (5) e o resultado experimental correspondente, obtido a partir dos dados do DSP.

Essas formas de onda, incluindo um detalhe transitório, mostram a boa correspondência entre a simulação e os resultados experimentais, confirmando a validade dos modelos

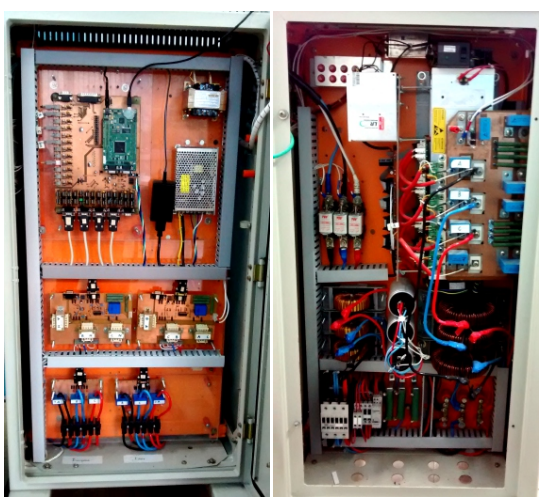

Fig. 6. Protótipo utilizado para obtenção dos resultados experimentais.

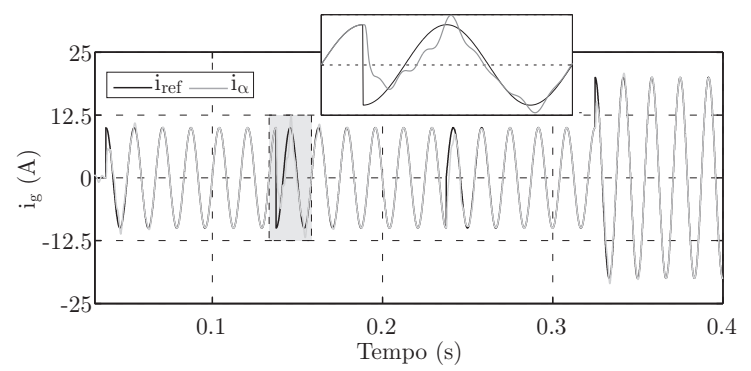

(a)

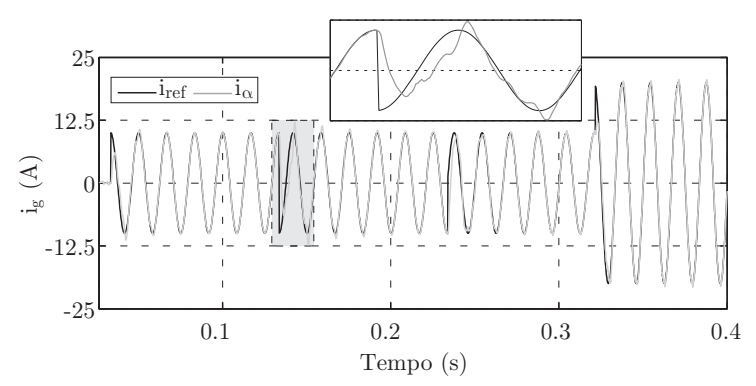

(b)

Fig. 7. Comparação do rastreamento de referência senoidal com mudanças de fase e amplitude: (a) simulação e (b) experimental. 
apresentados na Seção II, utilizados para orientar o GA na busca de ganhos de controle viáveis na prática. As mesmas conclusões são válidas para o eixo $\beta$.

As correntes trifásicas injetadas na rede, correspondentes ao teste mostrado na Figura 7, são dadas na Figura 8, com ênfase para dois dos transitórios. Os resultados confirmam experimentalmente a estabilidade do sistema em malha fechada e a boa qualidade na recuperação transitória e no regime permanente.

A Figura 9.a mostra as correntes trifásicas em regime permanente injetadas na rede. A Figura 9.b mostra a análise harmônica de uma dessas correntes, confirmando a compatibilidade com as exigências da norma IEEE 1547. A distorção harmônica total (THD) dessa forma de onda é de 2,2\%, atendendo aos limites prescritos por esta norma.

Para verificar experimentalmente a robustez do sistema com o controlador (15), a Figura 10 mostra um teste de mudança abrupta na indutância $L_{g}$, de $L_{\text {gmin }}$ para $L_{\text {gmax }}$. A estabilidade contra essa variação paramétrica está de acordo com o certificado teórico fornecido pelas LMIs na Seção IV.A.

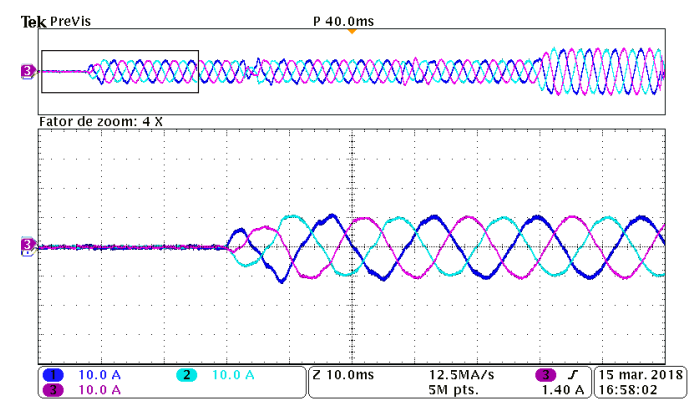

(a)

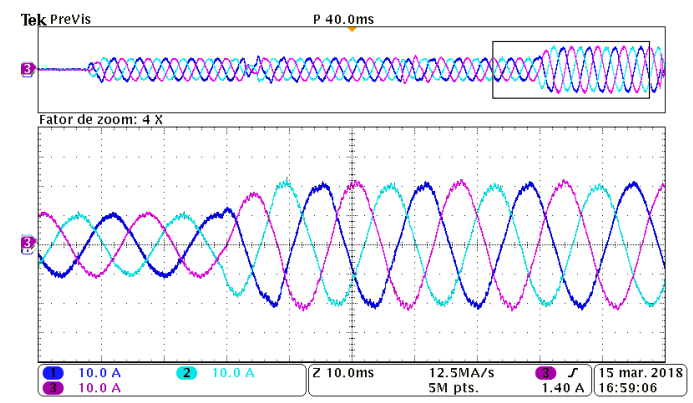

(b)

Fig. 8. Recuperações transitórias das correntes de rede trifásicas com controlador (15): (a) partida; (b) aumento de potência ativa.

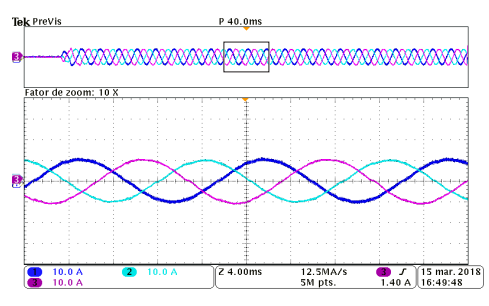

(a)

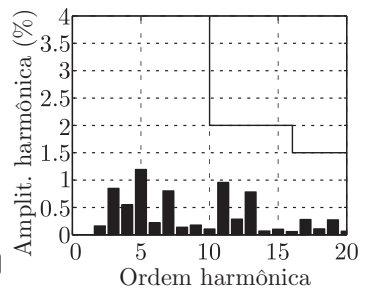

(b)
Fig. 9. Resultados experimentais para o controlador (15): (a) correntes de rede trifásicas, (b) espectro harmônico para a corrente no canal 1 e limites da norma IEEE 1547.

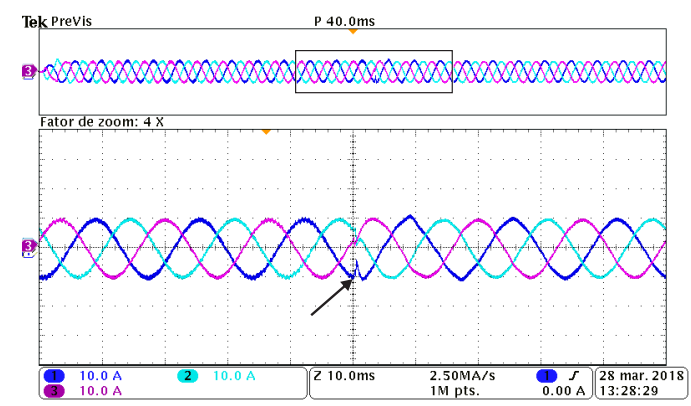

Fig. 10. Resultados experimentais: teste de robustez para uma variação abrupta da indutância de $L_{\text {gmin }}$ para $L_{\text {gmax }}$.

Para ilustrar a eficácia do procedimento de projeto proposto na Seção IV, o algoritmo foi executado 20 vezes, provendo, em todos os casos, controladores com estabilidade robusta certificada por (20) e resultados experimentais com padrão de transitório semelhante aos apresentados na Figura 8. Todos os resultados apresentaram regime permanente satisfatório, com THD abaixo do limite estabelecido pela a norma IEEE 1547 $(5 \%)$, indicando a boa qualidade das correntes injetadas na rede. A Tabela III apresenta a média e o desvio padrão normalizado pela média para os valores dos objetivos nestas execuções.

TABELA III

Média e Desvio Padrão dos Objetivos para Diferentes Execuções do Algoritmo

\begin{tabular}{c|c|c|c}
\hline \hline & $\sigma$ & $\varepsilon$ & $\gamma$ \\
\hline Média & 0,99736 & 1,66865 & 0,11524 \\
Desvio Padrão & $0,13 \%$ & $15,44 \%$ & $9,83 \%$ \\
\hline \hline
\end{tabular}

\section{A. Controlador por Realimentação Parcial de Estados}

Uma possível extensão do procedimento proposto, que confirma sua flexibilidade, é o projeto de controladores por realimentação parcial de estados. Esta é uma alternativa de controle importante, uma vez que permite implementar o controlador com um número menor de sensores, reduzindo os custos de implementação em comparação à realimentação total de estados.

Para ilustrar essa característica, os limites superior e inferior do segundo elemento do ganho, em (14), foram feitos iguais a zero, eliminando assim a necessidade de utilizar as medições das tensões nos capacitores do filtro LCL. O procedimento descrito na Figura 3 foi executado, levando aos ganhos de realimentação parcial de estados

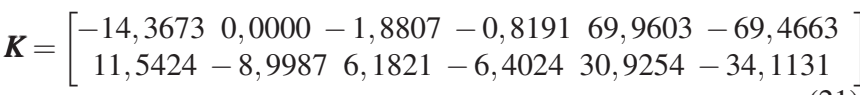

Note que o procedimento proposto fornece, neste caso, ganhos de controle robustos que não são alcançáveis por LMIs de síntese existentes, como as apresentadas em [15], [20], devido à necessidade de estruturas bloco diagonais para as matrizes que recuperam o ganho do controlador.

As correntes de rede, em regime permanente, são mostradas na Figura 11 .a. É possível confirmar que o sistema de malha fechada com controle por realimentação parcial de estados foi capaz de sintetizar correntes com boa qualidade, com THD de 3,4\%, também atendendo aos limites harmônicos da norma 
IEEE 1547, como mostrado na Figura 11.b. Por outro lado, ao utilizar a realimentação parcial de estados, embora seja possível eliminar sensores, o sistema apresenta recuperação transitória mais lenta, como ilustra a Figura 12. Respostas mais rápidas com realimentação parcial podem ser obtidas para outros conjuntos de parâmetros do filtro.

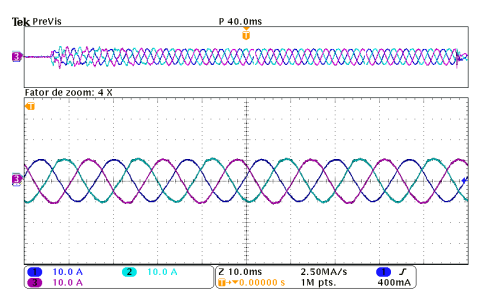

(a)

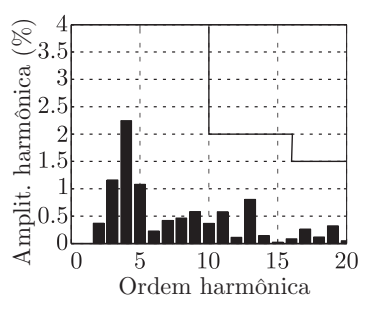

(b)
Fig. 11. Resultados experimentais para o controlador de realimentação parcial de estados (21): (a) correntes de rede trifásicas, (b) espectro harmônico e limites da norma IEEE 1547.

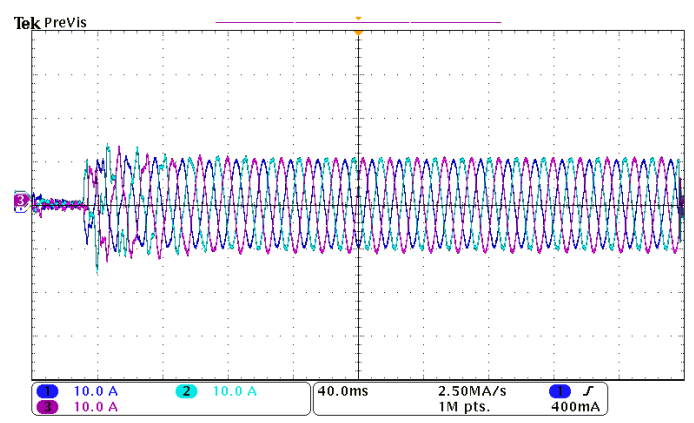

Fig. 12. Resultados transitório com o controlador de realimentação parcial de estados.

\section{CONCLUSÕES}

Este artigo fornece um novo procedimento de projeto para controladores de corrente aplicados a conversores conectados à rede por meio de filtros LCL. O procedimento é baseado em uma otimização multiobjetivo que usa um GA para encontrar os ganhos de controle, e LMIs para certificar a estabilidade robusta. As funções objetivo consideram estabilidade sob incertezas paramétricas, rastreamento de referências senoidais e rejeição de harmônicas, critérios que são de importância prática nesta aplicação. Dessa forma, o procedimento proposto permite acessar ganhos de controle que não são computáveis por meio de condições existentes de LMIs de síntese, como é o caso de ganhos de controle por realimentação parcial de estados. A boa correspondência entre resultados de simulação e experimentais indica que os modelos lineares utilizados aqui são adequados para orientar o GA, contribuindo para sua eficiência computacional. Os resultados experimentais demonstram robustez sob variações paramétricas na indutância da rede e confirmam a boa qualidade das correntes injetadas na rede, mostrando um bom rastreamento de referências senoidais, respostas transitórias adequadas e conteúdo harmônico que atende à norma IEEE 1547, indicando o procedimento proposto como interessante alternativa para projeto de controle robusto para conversores de potência.

\section{AGRADECIMENTOS}

O presente trabalho foi realizado com apoio da Coordenação de Aperfeiçoamento de Pessoal de Nível Superior - Brasil (CAPES/PROEX) - Código de Financiamento 001, INCT-GD, CNPq (465640/2014-1), CAPES (23038.000776/2017-54), FAPERGS (17/25510000517-1) e CNPq (Projeto 306197/2015-4).

\section{REFERÊNCIAS}

[1] G. Chiandussi, M. Codegone, S. Ferrero, F. Varesio, "Comparison of multi-objective optimization methodologies for engineering applications", Computers \& Mathematics with Applications, vol. 63, no. 5, pp. 912 - 942, Março 2012.

[2] R. Haupt, S. Haupt, Practical Genetic Algorithms, Wiley-Interscience publication, John Wiley, 2004.

[3] S. Durgadevi, M. G. Umamaheswari, "Analysis and design of single phase power factor correction with DC-DC SEPIC converter for fast dynamic response using genetic algorithm optimised PI controller", IET Circuits, Devices Systems, vol. 12, no. 2, pp. 164-174, Março 2018.

[4] F. H. Dupont, V. F. Montagner, J. R. Pinheiro, H. Pinheiro, S. V. G. Oliveira, A. Péres, "Comparison of linear quadratic controllers with stability analysis for DC-DC boost converters under large load range", Asian Journal of Control, vol. 15, no. 3, pp. 861-871, Maio 2013.

[5] R. Pena-Alzola, M. Liserre, F. Blaabjerg, M. Ordonez, Y. Yang, "LCL-Filter Design for Robust Active Damping in Grid-Connected Converters", IEEE Transactions on Industrial Informatics, vol. 10, no. 4, pp. 2192-2203, Novembro 2014.

[6] R. Teodorescu, M. Liserre, P. Rodríguez, Grid Converters for Photovoltaic and Wind Power Systems, Wiley - IEEE, John Wiley \& Sons, 2011.

[7] Standard for interconnecting distributed resources with electric power systems, IEEE Std. 1547 , Julho 2011.

[8] F. Blaabjerg, R. Teodorescu, M. Liserre, A. Timbus, "Overview of Control and Grid Synchronization for Distributed Power Generation Systems", IEEE Transactions on Industrial Electronics, vol. 53, no. 5, pp. 1398 -1409, Outubro 2006.

[9] B. Liu, Q. Wei, C. Zou, S. Duan, "Stability Analysis of LCL-Type Grid-Connected Inverter Under Single-Loop Inverter-Side Current Control With Capacitor Voltage Feedforward", IEEE Transactions on Industrial Informatics, vol. 14, no. 2, pp. 691-702, Fevereiro 2018.

[10] D. Pérez-Estévez, J. Doval-Gandoy, A. G. Yepes, O. López, F. Baneira, "Enhanced Resonant Current Controller for Grid-Connected Converters With LCL Filter", IEEE Transactions on Power Electronics, vol. 33, no. 5, pp. 3765-3778, Maio 2018.

[11] N. A. Windarko, O. A. Qudsi, A. Tjahjono, O. A. Dimas, M. H. Purnomo, "Optimized PI constant for current controller of grid connected inverter with LCL 
filter using Genetic Algorithm", in 2014 Makassar International Conference on Electrical Engineering and Informatics (MICEEI), pp. 9-13, Novembro 2014.

[12] E. Ebrahimzadeh, F. Blaabjerg, X. Wang, C. L. Bak, "Reducing Harmonic Instability and Resonance Problems in PMSG-Based Wind Farms", IEEE Journal of Emerging and Selected Topics in Power Electronics, vol. 6, no. 1, pp. 73-83, Março 2018.

[13] M. Liserre, A. Dell'Aquila, F. Blaabjerg, "Genetic algorithm-based design of the active damping for an LCL-filter three-phase active rectifier", Power Electronics, IEEE Transactions on, vol. 19, no. 1, pp. 76 - 86, Janeiro 2004.

[14] S. E. D. León-Aldaco, H. Calleja, J. A. Alquicira, "Metaheuristic Optimization Methods Applied to Power Converters: A Review", IEEE Transactions on Power Electronics, vol. 30, no. 12, pp. 6791-6803, Dezembro 2015.

[15] S. Boyd, L. El Ghaoui, E. Feron, V. Balakrishnan, Linear Matrix Inequalities in System and Control Theory, SIAM Studies in Applied Mathematics, Philadelphia, PA, 1994.

[16] L. A. Maccari, Jr., J. R. Massing, L. Schuch, C. Rech, H. Pinheiro, R. C. L. F. Oliveira, V. F. Montagner, "LMI-Based Control for Grid-Connected Converters With LCL Filters Under Uncertain Parameters", IEEE Transactions on Power Electronics, vol. 29, no. 7, pp. 3776-3785, Julho 2014.

[17] C. Scherer, " $\mathscr{H}_{\infty}$ control by state feedback: An iterative algorithm and characterization of high-gain occurence", Systems \& Control Letters, vol. 12, no. 5, pp. 383 - 391, 1989.

[18] M. V. Kothare, V. Balakrishnan, M. Morari, "Robust constrained model predictive control using linear matrix inequalities", Automatica, vol. 32, no. 10, pp. 1361 - 1379, Outubro 1996.

[19] R. C. L. F. Oliveira, P. L. D. Peres, "Parameterdependent LMIs in robust analysis: characterization of homogeneous polynomially parameter-dependent solutions via LMI relaxations", IEEE Transactions on Automatic Control, 2007.

[20] J. Daafouz, J. Bernussou, "Parameter dependent Lyapunov functions for discrete time systems with time varying parameter uncertainties", Systems \& Control Letters, vol. 43, no. 5, pp. 355-359, Agosto 2001.

[21] W. Duesterhoeft, M. W. Schulz, E. Clarke, "Determination of Instantaneous Currents and Voltages by Means of Alpha, Beta, and Zero Components", American Institute of Electrical Engineers, Transactions of the, vol. 70, no. 2, pp. 1248-1255, Julho 1951.

[22] K. J. Åström, B. Wittenmark, Adaptive Control, Addison-Wesley, Reading, MA, 1995, second edition.

[23] B. A. Francis, W. M. Wonham, "The internal model principle of control theory", Automatica, vol. 12, no. 5, pp. 457-465, Setembro 1976.

[24] L. A. Maccari, A. C. L. Santini, H. Pinheiro, R. C. L. F. de Oliveira, V. F. Montagner, "Robust optimal current control for grid-connected three-phase pulsewidth modulated converters", IET, Power Electronics, vol. 8, no. 8, pp. 1490-1499, Julho 2015.

[25] K. Deb, A. Pratap, S. Agarwal, T. Meyarivan, "A fast and elitist multiobjective genetic algorithm: NSGAII", IEEE Transactions on Evolutionary Computation, vol. 6, no. 2, pp. 182-197, Abril 2002.

[26] R. C. Dorf, R. H. Bishop, Modern control systems, 11 ed., Prentice Hall, Upper Saddle River, USA, 2008.

[27] R. Cardoso, R. F. de Camargo, H. Pinheiro, H. A. Gründling, "Kalman filter based synchronisation methods", Generation, Transmission Distribution, IET, vol. 2, no. 4, pp. 542-555, Julho 2008.

\section{BIOGRAFIAS}

Caio Ruviaro Dantas Osório Engenheiro eletricista (2015) e mestre em Engenharia Elétrica (2017) pela Universidade Federal de Santa Maria, Santa Maria, Brasil, onde atualmente realiza seu doutoramento. Suas áreas de interesse incluem controle aplicado a eletrônica de potência e controle robusto.

Gustavo Guilherme Koch Engenheiro eletricista (2013) e mestre em Engenharia Elétrica (2015) pela Universidade Federal de Santa Maria, Santa Maria, Brasil, onde atualmente realiza seu doutoramento. Suas áreas de interesse são: controle aplicado e controle robusto.

Iury Cleveston Graduado em Engenharia de Computação (2019) pela Universidade Federal de Santa Maria, Santa Maria. Suas áreas de interesse são: inteligência artificial e mineração de dados.

Lucas Cielo Borin Graduado em Engenharia de Computação (2018) pela Universidade Federal de Santa Maria, Santa Maria, Brasil, onde atualmente realiza o mestrado. Suas áreas de interesse são: microeletrônica e controle aplicado.

Fabrício Hoff Dupont Engenheiro de telecomunicação (2007) e mestre (2010) em Engenharia Elétrica pela Universidade de Blumenau, doutor (2014) em Engenharia Elétrica pela Universidade Federal de Santa Maria, Santa Maira, Brasil. É atualmente professor na Universidade Comunitária da Região de Chapecó. Suas áreas de interesse incluem eletrônica de potência, controle e otimização.

\section{Ricardo Coração de Leão Fontoura de Oliveira}

Engenheiro de computação (2001) pela Pontifícia Universidade Católica do Paraná, mestre (2003) e doutor (2006) em Engenharia Elétrica pela Universidade Estadual de Campinas, Campinas, Brasil. Atualmente é professor desta instituição. Suas áreas de interesse incluem análise de estabilidade e projeto de controladores para sistemas incertos.

Vinícius Foletto Montagner Engenheiro eletricista (1996) e mestre (2000) em Engenharia Elétrica pela Universidade Federal de Santa Maria, Santa Maria, Brasil, e doutor (2005) pela Universidade Estadual de Campinas. Atualmente é professor da Universidade Federal de Santa Maria. Suas áreas de interesse incluem estabilidade robusta, controle de sistemas incertos, otimização e controle aplicado. 